\title{
Supervised Data Classification via Max-min Separability
}

\author{
Adil M. Bagirov and Julien Ugon \\ CIAO, School of Information Technology and Mathematical Sciences \\ University of Ballarat \\ VIC 3353, Australia \\ a.bagirov@ballarat.edu.au, j.ugon@ballarat.edu.au
}

Summary. The problem of discriminating between the elements of two finite sets of points in $n$-dimensional space is a fundamental in supervised data classification. In practice, it is unlikely for the two sets to be linearly separable. In this paper we consider the problem of separating of two finite sets of points by means of piecewise linear functions. We prove that if these two sets are disjoint then they can be separated by a piecewise linear function and formulate the problem of finding the latter function as an optimization problem with an objective function containing max-min of linear functions. The differential properties of the objective function are studied and an algorithm for its minimization is developed. We present the results of numerical experiments with real world data sets. These results demonstrate the effectiveness of the proposed algorithm for separating two finite sets of points. They also demonstrate the effectiveness of an algorithm based on the concept of max-min separability for solving supervised data classification problems.

Key words: Supervised data classification, separability, nonconvex optimization, nonsmooth optimization.

\section{Introduction}

Supervised data classification is an important area in data mining. It has many applications in science, engineering, medicine etc. The aim of supervised data classification is to establish rules for the classification of some observations assuming that the classes of data are known. To find these rules, known training subsets of the given classes are used. During the last decades many algorithms have been proposed and studied to solve supervised data classification problems. One of the promising approaches to these problems is based on mathematical programming techniques. This approach has gained a great deal of attention over last years, see, for example, [AG02, Bag05, BRSY01, BRY00, BRY02, BB97, BB96, BM92, BM00, BFM99, Bur98, CM95, Man94, Man97, Tho02, Vap95]. 
There are different approaches for solving supervised data classification problems based on mathematical programming techniques. In one of them the use of mathematical programming techniques is carried out by reducing the classification problem to the problem of separation of two finite sets of points $A$ and $B$ in $n$-dimensional space. If $\operatorname{co} A \bigcap \operatorname{co} B=\emptyset$ then these two sets are linearly separable and there exists a hyperplane which separates these two sets. Linear programming techniques can be used to construct such a hyperplane. If the convex hulls of $A$ and $B$ intersect then linear programming techniques can be applied to obtain a hyperplane which minimizes some misclassification measure. Algorithms based on such an approach are developed in [BB96, BM92, CM95, Man94].

The paper [BM93] develops the concept of bilinear separability, where two sets are separated using two hyperplanes. The problem of finding of these hyperplanes is reduced to a certain bilinear programming problem. The paper [BM93] presents an algorithm for solving the latter problem.

In the paper [AG02] the concept of polyhedral separability was introduced. In this paper the case when $\operatorname{co} A \cap B=\emptyset$ was considered. The set $A$ is approximated by a polyhedral set. It is proved that the sets $A$ and $B$ are $h$ polyhedrally separable for some $h \leq|B|$, where $|B|$ is the cardinality of the set $B$. Thus in this case the sets $A$ and $B$ can be separated by a certain piecewise linear function. The authors introduce an error function which is nonconvex piecewise linear function. An algorithm for minimizing this function is proposed. The problem of the calculation of the descent direction in this algorithm is reduced to a certain linear programming problem.

The paper [Bag05] introduces the notion of max-min separability where two sets are separated by a piecewise linear function. Since any piecewise linear function can be represented as a max-min of linear functions we call it max-min separability. This approach can be considered as a generalization of the linear, bilinear and polyhedral separabilities.

The problem of max-min separability is reduced to a certain nonsmooth, nonconvex optimization problem. The objective function in this problem is represented as a sum of functions containing max-min of linear functions and it is a locally Lipschitz continuous. However this function is not Clarke regular and the calculation of its subgradient is a difficult task. Therefore methods of nonsmooth optimization based on subgradient information are not appropriate for solving max-min separability problems.

In this paper we develop an algorithm for solving max-min separability problems which uses only values of the objective function. This algorithm calculates a descent direction by evaluating the so-called discrete gradient of the objective function. The form of the objective function allows to significantly reduce the number of its evaluations during the computation of the discrete gradient. This is very important because each evaluations of the objective function for large data sets is expensive.

We carried out some numerical experiments using large scale data sets. We present their results and discuss them. 
The structure of this paper is as follows. Section 2 provides some preliminaries. In Section 3 the definition and some results related to the max-min separability are given. An algorithm for solving max-min separability problems is discussed in Section 4. Results of numerical experiments are presented in Section 5. Section 6 concludes the paper.

\section{Preliminaries}

In this section we present a brief review of the concepts of linear, bilinear and polyhedral separability.

\subsection{Linear separability}

Let $A$ and $B$ be given sets containing $m$ and $p n$-dimensional vectors, respectively:

$$
\begin{gathered}
A=\left\{a^{1}, \ldots, a^{m}\right\}, a^{i} \in \mathbb{R}^{n}, i=1, \ldots, m, \\
B=\left\{b^{1}, \ldots, b^{p}\right\}, b^{j} \in \mathbb{R}^{n}, j=1, \ldots, p .
\end{gathered}
$$

The sets $A$ and $B$ are linearly separable if there exists a hyperplane $\{x, y\}$, with $x \in \mathbb{R}^{n}, y \in \mathbb{R}^{1}$ such that

1) for any $j=1, \ldots, m$

$$
\left\langle x, a^{j}\right\rangle-y<0
$$

2) for any $k=1, \ldots, p$

$$
\left\langle x, b^{k}\right\rangle-y>0 \text {. }
$$

The sets $A$ and $B$ are linearly separable if and only if $\operatorname{co} A \bigcap \operatorname{co} B=\emptyset$.

In practice, it is unlikely for the two sets to be linearly separable. Therefore it is important to find a hyperplane which minimizes some misclassification cost. In the paper [BM92] the problem of finding this hyperplane is formulated as the following optimization problem:

$$
\text { minimize } f(x, y) \text { subject to }(x, y) \in \mathbb{R}^{n+1}
$$

where

$$
f(x, y)=\frac{1}{m} \sum_{i=1}^{m} \max \left(0,\left\langle x, a^{i}\right\rangle-y+1\right)+\frac{1}{p} \sum_{j=1}^{p} \max \left(0,-\left\langle x, b^{j}\right\rangle+y+1\right)
$$

is an error function. Here $\langle\cdot, \cdot\rangle$ stands for the scalar product in $\mathbb{R}^{n}$. The authors describe an algorithm for solving problem (1). They show that the problem (1) is equivalent to the following linear program:

$$
\operatorname{minimize} \frac{1}{m} \sum_{i=1}^{m} t_{i}+\frac{1}{p} \sum_{j=1}^{p} z_{j}
$$


subject to

$$
\begin{gathered}
t_{i} \geq\left\langle x, a^{i}\right\rangle-y+1, \quad i=1, \ldots, m, \\
z_{j} \geq-\left\langle x, b^{j}\right\rangle+y+1, \quad j=1, \ldots, p, \\
t \geq 0, \quad z \geq 0
\end{gathered}
$$

where $t_{i}$ is nonnegative and represents the error for the point $a^{i} \in A$ and $z_{j}$ is nonnegative and represents the error for the point $b^{j} \in B$.

The sets $A$ and $B$ are linearly separable if and only if $f^{*}=f\left(x^{*}, y_{*}\right)=0$ where $\left(x^{*}, y_{*}\right)$ is the solution to the problem (1). It is proved that the trivial solution $x=0$ cannot occur.

\subsection{Bilinear separability}

The concept of bilinear separability was introduced in [BM93]. In this approach two sets are separated using two hyperplanes. We again assume that $A$ and $B$ are given sets containing $m$ and $p n$-dimensional vectors, respectively.

Definition 1. (see [BM93]). The sets $A$ and $B$ are bilinear separable if and only if there exist two hyperplanes $\left(x^{1}, y_{1}\right)$ and $\left(x^{2}, y_{2}\right)$ such that at least one of the following conditions holds:

1. For any $j=1, \ldots, m$

$$
\left\langle x^{l}, a^{j}\right\rangle-y_{l}<0, \quad l=1,2
$$

and for any $k=1, \ldots, p$ there exists $l \in\{1,2\}$ such that

$$
\left\langle x^{l}, b^{k}\right\rangle-y_{l}>0 \text {. }
$$

2. For any $k=1, \ldots, p$

$$
\left\langle x^{l}, b^{k}\right\rangle-y_{l}<0, \quad l=1,2
$$

and for any $j=1, \ldots, m$ there exists $l \in\{1,2\}$ such that

$$
\left\langle x^{l}, a^{j}\right\rangle-y_{l}>0 \text {. }
$$

3. For any $j=1, \ldots, m$ either

$$
\left\langle x^{l}, a^{j}\right\rangle-y_{l}<0, \quad l=1,2
$$

or

$$
\left\langle-x^{l}, a^{j}\right\rangle+y_{l}<0, \quad l=1,2
$$

and for any $k=1, \ldots, p$ either

$$
\left\langle x^{1}, b^{k}\right\rangle-y_{1}<0, \quad\left\langle-x^{2}, b^{k}\right\rangle+y_{2}<0
$$

or

$$
\left\langle-x^{1}, b^{k}\right\rangle+y_{1}<0, \quad\left\langle x^{2}, b^{k}\right\rangle-y_{2}>0 .
$$


We reformulate Definition 1 using max and min statements.

Definition 2. The sets $A$ and $B$ are bilinear separable if and only if there exist two hyperplanes $\left(x^{1}, y_{1}\right)$ and $\left(x^{2}, y_{2}\right)$ such that at least one of the following conditions holds:

1. For any $j=1, \ldots, m$

$$
\max _{l=1,2}\left\{\left\langle x^{l}, a^{j}\right\rangle-y_{l}\right\}<0
$$

and for any $k=1, \ldots, p$

$$
\max _{l=1,2}\left\{\left\langle x^{l}, b^{k}\right\rangle-y_{l}\right\}>0 .
$$

2. For any $k=1, \ldots, p$

$$
\max _{l=1,2}\left\{\left\langle x^{l}, b^{k}\right\rangle-y_{l}\right\}<0
$$

and for any $j=1, \ldots, m$

$$
\max _{l=1,2}\left\{\left\langle x^{l}, a^{j}\right\rangle-y_{l}\right\}>0 .
$$

3. For any $j=1, \ldots, m$,

$$
\begin{array}{r}
\max \left[\min \left\{\left\langle x^{1}, a^{j}\right\rangle-y_{1},-\left\langle x^{2}, a^{j}\right\rangle+y_{2}\right\}, \min \left\{-\left\langle x^{1}, a^{j}\right\rangle+y_{1},\left\langle x^{2}, a^{j}\right\rangle-y_{2}\right\}\right] \\
<0
\end{array}
$$

and for any $k=1, \ldots, p$,

$$
\begin{array}{r}
\max \left[\min \left\{\left\langle x^{1}, b^{k}\right\rangle-y_{1},-\left\langle x^{2}, b^{k}\right\rangle+y_{2}\right\}, \min \left\{-\left\langle x^{1}, b^{k}\right\rangle+y_{1},\left\langle x^{2}, b^{k}\right\rangle-y_{2}\right\}\right] \\
>0 .
\end{array}
$$

The problem of bilinear separability is reduced to a certain bilinear programming problem and the paper [BM93] presents an algorithm for its solution.

\subsection{Polyhedral separability}

The concept of $h$-polyhedral separability was developed in [AG02]. The sets $A$ and $B$ are $h$-polyhedrally separable if there exists a set of $h$ hyperplanes $\left\{x^{i}, y_{i}\right\}$, with

$$
x^{i} \in \mathbb{R}^{n}, y_{i} \in \mathbb{R}^{1}, i=1, \ldots, h
$$

such that

1) for any $j=1, \ldots, m$ and $i=1, \ldots, h$

$$
\left\langle x^{i}, a^{j}\right\rangle-y_{i}<0,
$$


2) for any $k=1, \ldots, p$ there exists at least one $i \in\{1, \ldots, h\}$ such that

$$
\left\langle x^{i}, b^{k}\right\rangle-y_{i}>0 .
$$

It is proved in [AG02] that the sets $A$ and $B$ are $h$-polyhedrally separable, for some $h \leq p$ if and only if

$$
\operatorname{co} A \bigcap B=\emptyset \text {. }
$$

Figure 1 presents one example of polyhedral separability.

The problem of polyhedral separability of the sets $A$ and $B$ is reduced to the following problem:

$$
\text { minimize } f(x, y) \text { subject to }(x, y) \in \mathbb{R}^{(n+1) \times h}
$$

where

$$
\begin{gathered}
f(x, y)=\frac{1}{m} \sum_{j=1}^{m} \max \left[0, \max _{1 \leq i \leq h}\left\{\left\langle x^{i}, a^{j}\right\rangle-y_{i}+1\right\}\right]+ \\
\frac{1}{p} \sum_{k=1}^{p} \max \left[0, \min _{1 \leq i \leq h}\left\{-\left\langle x^{i}, b^{k}\right\rangle+y_{i}+1\right\}\right]
\end{gathered}
$$

is an error function. Note that this function is a nonconvex piecewise linear function. It is proved that $x^{i}=0, i=1, \ldots, h$ cannot be the optimal solution. Let $\left\{\bar{x}^{i}, \bar{y}_{i}\right\}, i=1, \ldots, h$ be a global solution to the problem (2). The sets $A$ and $B$ are $h$-polyhedrally separable if and only if $f(\bar{x}, \bar{y})=0$. If there exists a nonempty set $\bar{I} \subset\{1, \ldots, h\}$ such that $x^{i}=0, i \in \bar{I}$, then the sets $A$ and $B$ are $(h-|\bar{I}|)$-polyhedrally separable. In [AG02] an algorithm for solving problem (2) is developed. The calculation of the descent direction at each iteration of this algorithm is reduced to a certain linear programming problem.

The advantage of this technique is that it does not restrict the search to only a convex polyhedron, and thus allows both the sets $A$ and $B$ to be nonconvex. One disadvantage, however, is that it only considers the sets separately.

\section{Max-min separability}

In many practical applications two sets are not linearly, bilinearly or polyhedrally separable. Figure 2 presents one such case. In this case two sets are separable with more complicated piecewise linear function.

In this section we describe the concept of max-min separability and introduce an error function (see $[\mathrm{Bag} 05]$ ). 


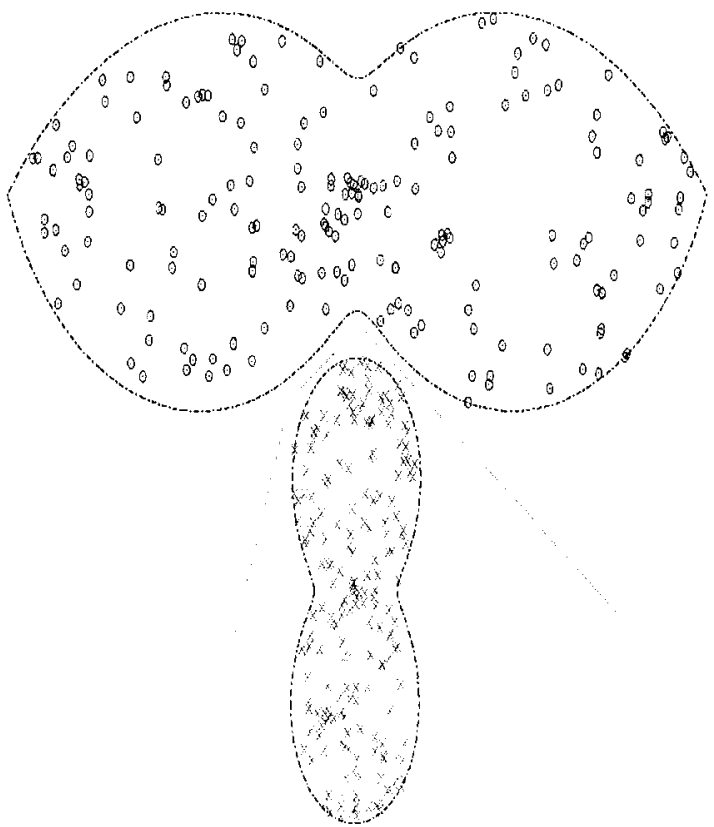

Fig. 1. Polyhedral separability.

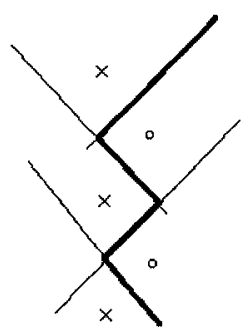

Fig. 2. The sets $A$ and $B$ are separated by a piecewise linear function.

\subsection{Definition and properties}

Let $H=\left\{h_{1}, \ldots, h_{l}\right\}$, where $h_{j}=\left\{x^{j}, y_{j}\right\}, j=1, \ldots, l$ with $x^{j} \in \mathbb{R}^{n}, y_{j} \in$ $\mathbb{R}^{1}$, be a finite set of hyperplanes. Let $J=\{1 \ldots, l\}$. Consider any partition 
of this set $J^{r}=\left\{J_{1}, \ldots, J_{r}\right\}$ such that

$$
J_{k} \neq \emptyset, k=1, \ldots, r, \quad J_{k} \bigcap J_{j}=\emptyset, \quad \bigcup_{k=1}^{r} J_{k}=J .
$$

Let $I=\{1, \ldots, r\}$. A particular partition $J^{r}=\left\{J_{1}, \ldots, J_{r}\right\}$ of the set $J$ defines the following max-min-type function:

$$
\varphi(z)=\max _{i \in I} \min _{j \in J_{i}}\left\{\left\langle x^{j}, z\right\rangle-y_{j}\right\}, z \in \mathbb{R}^{n} .
$$

In Figure 3 two sets are max-min separable.

Let $A, B \subset \mathbb{R}^{n}$ be given disjoint sets, that is $A \cap B=\emptyset$.

Definition 3. The sets $A$ and $B$ are max-min separable if there exist a finite number of hyperplanes $\left\{x^{j}, y_{j}\right\}$ with $x^{j} \in \mathbb{R}^{n}, y_{j} \in \mathbb{R}^{1}, j \in J=\{1, \ldots, l\}$ and a partition $J^{r}=\left\{J_{1}, \ldots, J_{r}\right\}$ of the set $J$ such that

1) for all $i \in I$ and $a \in A$

$$
\min _{j \in J_{i}}\left\{\left\langle x^{j}, a\right\rangle-y_{j}\right\}<0
$$

2) for any $b \in B$ there exists at least one $i \in I$ such that

$$
\min _{j \in J_{i}}\left\{\left\langle x^{j}, b\right\rangle-y_{j}\right\}>0 \text {. }
$$

Remark 1. It follows from Definition 3 that if the sets $A$ and $B$ are max-min separable then $\varphi(a)<0$ for any $a \in A$ and $\varphi(b)>0$ for any $b \in B$, where the function $\varphi$ is defined by (3). Thus the sets $A$ and $B$ can be separated by a function represented as a max-min of linear functions. Therefore this kind of separability is called a max-min separability.

Remark 2. Linear and polyhedral separability can be considered as particular cases of the max-min separability. If $I=\{1\}$ and $J_{1}=\{1\}$ then we have the linear separability and if $I=\{1, \ldots, h\}$ and $J_{i}=\{i\}, i \in I$ we obtain the $h$-polyhedral separability.

Remark 3. Bilinear separability can also be considered as particular case of the max-min separability. It follows from Definition 2 that the bilinear separability of two sets $A$ and $B$ coincides with one of the following cases:

1. The sets $A$ and $B$ are 2-polyhedrally separable and $\operatorname{co} A \cap B=\emptyset$;

2. The sets $A$ and $B$ are 2-polyhedrally separable and co $B \cap A=\emptyset$;

3. The sets $A$ and $B$ are max-min separable with the following hyperplanes:

$$
\left\{\left(x^{1}, y_{1}\right),\left(-x^{1},-y_{1}\right),\left(x^{2}, y_{2}\right),\left(-x^{2},-y_{2}\right\}\right.
$$

In this case $I=\{1,2\}$ and $J_{1}=\{1,4\}, \quad J_{2}=\{2,3\}$. Thus the bilinear separable sets are also max-min separable. 


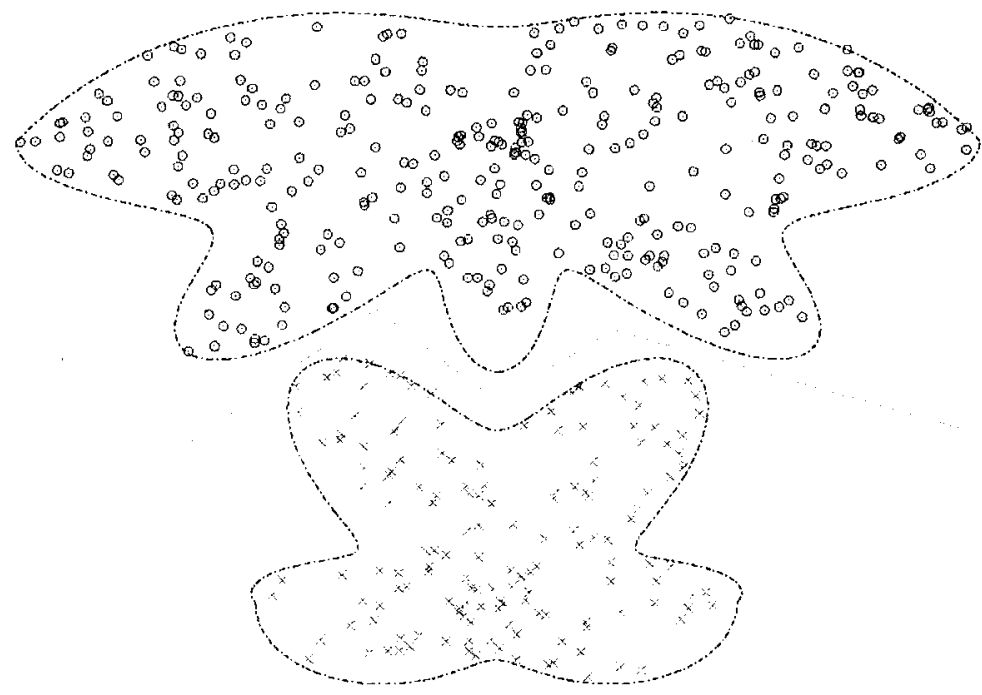

Fig. 3. Max-min separability.

Proposition 1. (see [Bag05]). The sets $A$ and $B$ are max-min separable if and only if there exists a set of hyperplanes $\left\{x^{j}, y_{j}\right\}$ with $x^{j} \in \mathbb{R}^{n}, y_{j} \in$ $\mathbb{R}^{1}, j \in J$ and a partition $J^{r}=\left\{J_{1}, \ldots, J_{r}\right\}$ of the set $J$ such that

1) for any $i \in I$ and $a \in A$

$$
\min _{j \in J_{i}}\left\{\left\langle x^{j}, a\right\rangle-y_{j}\right\} \leq-1
$$

2) for any $b \in B$ there exists at least one $i \in I$ such that

$$
\min _{j \in J_{i}}\left\{\left\langle x^{j}, b\right\rangle-y_{j}\right\} \geq 1 \text {. }
$$

Proof. Sufficiency is straightforward.

Necessity. Since $A$ and $B$ are max-min separable there exists a set of hyperplanes $\left\{\bar{x}^{j}, \bar{y}_{j}\right\}$ with $\bar{x}^{j} \in \mathbb{R}^{n}, \bar{y}_{j} \in \mathbb{R}^{1}, j \in J$, a partition $J^{r}$ of the set $J$ and numbers $\delta_{1}>0, \delta_{2}>0$ such that

$$
\max _{a \in A} \max _{i \in I} \min _{j \in J_{i}}\left\{\left\langle\bar{x}^{j}, a\right\rangle-\bar{y}_{j}\right\}=-\delta_{1}
$$

and

$$
\min _{b \in B} \max _{i \in I} \min _{j \in J_{i}}\left\{\left\langle\bar{x}^{j}, b\right\rangle-\bar{y}_{j}\right\}=\delta_{2} \text {. }
$$

We put $\delta=\min \left\{\delta_{1}, \delta_{2}\right\}>0$. Then we have

$$
\max _{i \in I} \min _{j \in J_{i}}\left\{\left\langle\bar{x}^{j}, a\right\rangle-\bar{y}_{j}\right\} \leq-\delta, \forall a \in A,
$$




$$
\max _{i \in I} \min _{j \in J_{i}}\left\{\left\langle\bar{x}^{j}, b\right\rangle-\bar{y}_{j}\right\} \geq \delta, \forall b \in B
$$

We consider the new set of hyperplanes $\left\{x^{j}, y_{j}\right\}$ with $x^{j} \in \mathbb{R}^{n}, y_{j} \in \mathbb{R}^{1}, j \in$ $J$, defined as follows:

$$
\begin{aligned}
& x^{j}=\bar{x}^{j} / \delta, j \in J, \\
& y^{j}=\bar{y}^{j} / \delta, j \in J .
\end{aligned}
$$

Then it follows from (4) and (5) that

$$
\begin{gathered}
\max _{i \in I} \min _{j \in J_{i}}\left\{\left\langle x^{j}, a\right\rangle-y_{j}\right\} \leq-1, \quad \forall a \in A, \\
\max _{i \in I} \min _{j \in J_{i}}\left\{\left\langle x^{j}, b\right\rangle-y_{j}\right\} \geq 1, \quad \forall b \in B,
\end{gathered}
$$

which completes the proof.

Proposition 2. (see [Bag05]). The sets $A$ and $B$ are max-min separable if and only if there exists a piecewise linear function separating them.

Proof. Since max-min of linear functions is piecewise linear function the necessity is straightforward.

Sufficiency. It is known that any piecewise linear function can be represented as a max-min of linear functions of the form (3) (see [BKS95]). Then we get that there exists max-min of linear functions that separates the sets $A$ and $B$ which in its turn means that these sets are max-min separable.

Remark 4. It follows from Proposition (2) that the notions of max-min and piecewise linear separability are equivalent.

Proposition 3. (see [Bag05]). Assume that the set $A$ can be represented as a union of sets $A_{i}, i=1, \ldots, q$ :

$$
A=\bigcup_{i=1}^{q} A_{i}
$$

and for any $i=1, \ldots, q$

$$
B \bigcap \operatorname{co} A_{i}=\emptyset
$$

Then the sets $A$ and $B$ are max-min separable.

Proof. It follows from (6) that $b \notin \operatorname{co} A_{i}$ for all $b \in B$ and $i \in\{1, \ldots, q\}$. Then, for each $b \in B$ and $i \in\{1, \ldots, q\}$ there exists a hyperplane $\left\{x^{i}(b), y_{i}(b)\right\}$ separating $b$ from the set $\operatorname{co} A_{i}$, that is

$$
\left\langle x^{i}(b), b\right\rangle-y_{i}(b)>0
$$




$$
\left\langle x^{i}(b), a\right\rangle-y_{i}(b)<0, \quad \forall a \in \operatorname{co} A_{i}, i=1, \ldots, q .
$$

Then we have

$$
\min _{i=1, \ldots, q}\left\{\left\langle x^{i}(b), b\right\rangle-y_{i}(b)\right\}>0
$$

and

$$
\min _{i=1, \ldots, q}\left\{\left\langle x^{i}(b), a\right\rangle-y_{i}(b)\right\}<0, \quad \forall a \in A
$$

Thus we obtain that for any $b^{j} \in B, j=1, \ldots, p$ there exists a set of $q$ hyperplanes $\left\{x^{i}\left(b^{j}\right), y_{i}\left(b^{j}\right)\right\}, i=1, \ldots, q$ such that

$$
\min _{i=1, \ldots, q}\left\{\left\langle x^{i}\left(b^{j}\right), b^{j}\right\rangle-y_{i}\left(b^{j}\right)\right\}>0
$$

and

$$
\min _{i=1, \ldots, q}\left\{\left\langle x^{i}\left(b^{j}\right), a\right\rangle-y_{i}\left(b^{j}\right)\right\}<0, \quad \forall a \in A .
$$

Consequently we have $p q$ hyperplanes

$$
\left\{x^{i}\left(b^{j}\right), y_{i}\left(b^{j}\right)\right\}, i=1, \ldots, q, j=1, \ldots, p .
$$

The set of these hyperplanes can be rewritten as follows:

$$
\begin{gathered}
H=\left\{h_{1}, \ldots, h_{l}\right\}, \quad h_{i+(j-1) q}=\left\{x^{i}\left(b^{j}\right), y_{i}\left(b^{j}\right)\right\}, \\
i=1, \ldots, q, j=1, \ldots, p, l=p q .
\end{gathered}
$$

Let $J=\{1, \ldots, l\}, I=\{1, \ldots, p\}$ and

$$
\bar{x}^{i+(j-1) q}=x^{i}\left(b^{j}\right), \bar{y}_{i+(j-1) q}=y_{i}\left(b^{j}\right), i=1, \ldots, q, j=1, \ldots, p .
$$

Consider the following partition of the set $J$ :

$$
J^{p}=\left\{J_{1}, \ldots, J_{p}\right\}, J_{k}=\{(k-1) q+1, \ldots, k q\}, k=1, \ldots, p .
$$

It follows from (7) and (8) that for all $k \in I$ and $a \in A$

$$
\min _{j \in J_{k}}\left\{\left\langle\bar{x}^{j}, a\right\rangle-\bar{y}_{j}\right\}<0
$$

and for any $b \in B$ there exists at least one $k \in I$ such that

$$
\min _{j \in J_{k}}\left\{\left\langle\bar{x}^{j}, b\right\rangle-\bar{y}_{j}\right\}>0
$$

which means that the sets $A$ and $B$ are max-min separable.

Corollary 1. (see [Bag05]). The sets $A$ and $B$ are max-min separable if and only if they are disjoint: $A \cap B=\emptyset$.

Proof. Necessity is straightforward.

Sufficiency. The set $A$ can be represented as a union of its own points. Since the sets $A$ and $B$ are disjoint the condition (6) is satisfied. Then the proof of the corollary follows from Proposition 3. 
In the next proposition we show that in most cases the number of hyperlanes necessary for the max-min separation of the sets $A$ and $B$ is limited.

Proposition 4. (see [Bag05]). Assume that the set $A$ can be represented as a union of sets $A_{i}, i=1, \ldots, q$ and the set $B$ as a union of sets $B_{j}, j=1, \ldots, d$ such that

$$
A=\bigcup_{i=1}^{q} A_{i}, \quad B=\bigcup_{j=1}^{d} B_{j}
$$

and

$$
\operatorname{co}_{i} \bigcap \operatorname{co} B_{j}=\emptyset \text { for all } i=1, \ldots, q, j=1, \ldots, d \text {. }
$$

Then the number of hyperplanes necessary for the separation of the sets $A$ and $B$ is at most $q \cdot d$.

Proof. Let $i \in\{1, \ldots, q\}$ and $j \in\{1, \ldots, d\}$ be any fixed indices. Since $\operatorname{co} A_{i} \bigcap \operatorname{co} B_{j}=\emptyset$ there exists a hyperplane $\left\{x^{i j}, y_{i j}\right\}$ with $x^{i j} \in \mathbb{R}^{n}, y_{i j} \in \mathbb{R}^{1}$ such that

$$
\left\langle x^{i j}, a\right\rangle-y_{i j}<0 \quad \forall a \in \operatorname{co} A_{i}
$$

and

$$
\left\langle x^{i j}, b\right\rangle-y_{i j}>0 \quad \forall b \in \operatorname{co} B_{j} .
$$

Consequently for any $j \in\{1, \ldots, d\}$ there exists a set of hyperplanes $\left\{x^{i j}, y_{i j}\right\}$, $i=1, \ldots, q$ such that

$$
\min _{i=1, \ldots, q}\left\langle x^{i j}, b\right\rangle-y_{i j}>0, \forall b \in B_{j}
$$

and

$$
\min _{i=1, \ldots, q}\left\langle x^{i j}, a\right\rangle-y_{i j}<0, \forall a \in A .
$$

Thus we get a system of $l=d q$ hyperplanes:

$$
H=\left\{h_{1}, \ldots, h_{l}\right\}
$$

where $h_{i+(j-1) q}=\left\{x^{i j}, y_{i j}\right\}, i=1, \ldots, q, j=1, \ldots, d$. Let $J=\{1, \ldots, l\}$, $I=\{1, \ldots, d\}$ and

$$
\bar{x}^{i+(j-1) q}=x^{i j}, \bar{y}_{i+(j-1) q}=y_{i j}, i=1, \ldots, q, j=1, \ldots, d .
$$

Consider the following partition of the set $J$ :

$$
J^{d}=\left\{J_{1}, \ldots, J_{d}\right\}, J_{k}=\{(k-1) q+1, \ldots, k q\}, k=1, \ldots, d .
$$

It follows from (10) and (11) that for all $k \in I$ and $a \in A$

$$
\min _{j \in J_{k}}\left\{\left\langle\bar{x}^{j}, a\right\rangle-\bar{y}_{j}\right\}<0
$$


and for any $b \in B$ there exists at least one $k \in I$ such that

$$
\min _{j \in J_{k}}\left\{\left\langle\bar{x}^{j}, b\right\rangle-\bar{y}_{j}\right\}>0
$$

that is the sets $A$ and $B$ are max-min separable with at most $q \cdot d$ hyperplanes.

Remark 5. The only cases where the number of hyperplanes necessary is large are when the sets $A_{i}$ and $B_{j}$ contain a very small number of points. This situation appears only in the particular case where the distribution of the points is like a "chessboard".

\subsection{Error function}

Given any set of hyperplanes $\left\{x^{j}, y_{j}\right\}, j \in J=\{1, \ldots, l\}$ with $x^{j} \in \mathbb{R}^{n}, y_{j} \in$ $\mathbb{R}^{1}$ and a partition $J^{r}=\left\{J_{1}, \ldots, J_{r}\right\}$ of the set $J$, we say that a point $a \in A$ is well separated from the set $B$ if the following condition is satisfied:

$$
\max _{i \in I} \min _{j \in J_{i}}\left\{\left\langle x^{j}, a\right\rangle-y_{j}\right\}+1 \leq 0
$$

Then we can define the separation error for a point $a \in A$ as follows:

$$
\max \left[0, \max _{i \in I} \min _{j \in J_{i}}\left\{\left\langle x^{j}, a\right\rangle-y_{j}+1\right\}\right] \text {. }
$$

Analogously, a point $b \in B$ is said to be well separated from the set $A$ if the following condition is satisfied:

$$
\min _{i \in I} \max _{j \in J_{i}}\left\{-\left\langle x^{j}, b\right\rangle+y_{j}\right\}+1 \leq 0 .
$$

Then the separation error for a point $b \in B$ can be written as

$$
\max \left[0, \min _{i \in I} \max _{j \in J_{i}}\left\{-\left\langle x^{j}, b\right\rangle+y_{j}+1\right\}\right] .
$$

Thus, an averaged error function can be defined as

$$
\begin{gathered}
f(x, y)=(1 / m) \sum_{k=1}^{m} \max \left[0, \max _{i \in I} \min _{j \in J_{i}}\left\{\left\langle x^{j}, a^{k}\right\rangle-y_{j}+1\right\}\right] \\
+(1 / p) \sum_{t=1}^{p} \max \left[0, \min _{i \in I} \max _{j \in J_{i}}\left\{-\left\langle x^{j}, b^{t}\right\rangle+y_{j}+1\right\}\right]
\end{gathered}
$$

where $x=\left(x^{1}, \ldots, x^{l}\right) \in \mathbb{R}^{l \times n}, y=\left(y_{1}, \ldots, y_{l}\right) \in \mathbb{R}^{l}$. It is clear that $f(x, y) \geq$ 0 for all $x \in \mathbb{R}^{l \times n}$ and $y \in \mathbb{R}^{l}$. 
Proposition 5. (see [Bag05]). The sets $A$ and $B$ are max-min separable if and only if there exists a set of hyperplanes $\left\{x^{j}, y_{j}\right\}, j \in J=\{1, \ldots, l\}$ and a partition $J^{r}=\left\{J_{1}, \ldots, J_{r}\right\}$ of the set $J$ such that $f(x, y)=0$.

Proof. Necessity. Assume that the sets $A$ and $B$ are max-min separable. Then it follows from Proposition 1 that there exists a set of hyperplanes $\left\{x^{j}, y_{j}\right\}, j \in$ $J$ and a partition $J^{r}=\left\{J_{1}, \ldots, J_{r}\right\}$ of the set $J$ such that

$$
\min _{j \in J_{i}}\left\{\left\langle x^{j}, a\right\rangle-y_{j}\right\} \leq-1, \forall a \in A, i \in I=\{1, \ldots, r\}
$$

and for any $b \in B$ there exists at least one $t \in I$ such that

$$
\min _{j \in J_{t}}\left\{\left\langle x^{j}, b\right\rangle-y_{j}\right\} \geq 1 \text {. }
$$

Consequently we have

$$
\begin{aligned}
& \max _{i \in I} \min _{j \in J_{i}}\left\{\left\langle x^{j}, a\right\rangle-y_{j}+1\right\} \leq 0, \quad \forall a \in A, \\
& \min _{i \in I} \max _{j \in J_{i}}\left\{-\left\langle x^{j}, b\right\rangle+y_{j}+1\right\} \leq 0, \quad \forall b \in B .
\end{aligned}
$$

Then from the definition of the error function we obtain that $f(x, y)=0$.

Sufficiency. Assume that there exist a set of hyperplanes $\left\{x^{j}, y_{j}\right\}, j \in J=$ $\{1, \ldots, l\}$ and a partition $J^{r}=\left\{J_{1}, \ldots, J_{r}\right\}$ of the set $J$ such that $f(x, y)=0$. Then from the definition of the error function $f$ we immediately get that the inequalities (15) and (16) are satisfied, that is the sets $A$ and $B$ are max-min separable.

Proposition 6. (see [Bag05]). Assume that the sets $A$ and $B$ are max-min separable with a set of hyperplanes $\left\{x^{j}, y_{j}\right\}, j \in J=\{1, \ldots, l\}$ and a partition $J^{r}=\left\{J_{1}, \ldots, J_{r}\right\}$ of the set $J$. Then

1) $x^{j}=0, j \in J$ cannot be an optimal solution;

2) if

(a) for any $t \in I$ there exists at least one $b \in B$ such that

$$
\max _{j \in J_{t}}\left\{-\left\langle x^{j}, b\right\rangle+y_{j}+1\right\}=\min _{i \in I} \max _{j \in J_{i}}\left\{-\left\langle x^{j}, b\right\rangle+y_{j}+1\right\},
$$

(b) there exists $\tilde{J}=\left\{\tilde{J}_{1}, \ldots, \tilde{J}_{r}\right\}$ such that $\tilde{J}_{t} \subset J_{t}, \forall t \in I, \tilde{J}_{t}$ is nonempty at least for one $t \in I$ and $x^{j}=0$ for any $j \in \tilde{J}_{t}, t \in I$.

Then the sets $A$ and $B$ are max-min separable with a set of hyperplanes $\left\{x^{j}, y_{j}\right\}, j \in J^{0}$ and a partition $\bar{J}=\left\{\bar{J}_{1}, \ldots, \bar{J}_{r}\right\}$ of the set $J^{0}$ where

$$
\bar{J}_{t}=J_{t} \backslash \tilde{J}_{t}, t \in I \text { and } J^{0}=\bigcup_{i=1}^{r} \bar{J}_{i} .
$$


Proof. 1) Since the sets $A$ and $B$ are max-min separable we get from Proposition 5 that $f(x, y)=0$. If $x^{j}=0, j \in J$ then it follows from (14) that for any $y \in \mathbb{R}^{l}$

$$
\begin{gathered}
f(0, y)=(1 / m) \sum_{k=1}^{m} \max \left[0, \max _{i \in I} \min _{j \in J_{i}}\left\{-y_{j}+1\right\}\right] \\
+(1 / p) \sum_{t=1}^{p} \max \left[0, \min _{i \in I} \max _{j \in J_{i}}\left\{y_{j}+1\right\}\right] .
\end{gathered}
$$

We denote

$$
R=\max _{i \in I} \min _{j \in J_{i}}\left\{-y_{j}\right\}
$$

Then we have

$$
\min _{i \in I} \max _{j \in J_{i}} y_{j}=-\max _{i \in I} \min _{j \in J_{i}}\left\{-y_{j}\right\}=-R
$$

Thus

$$
f(0, y)=\max [0, R+1]+\max [0,-R+1] .
$$

It is clear that

$$
\max [0, R+1]+\max [0,-R+1]= \begin{cases}-R+1 & \text { if } R \leq-1 \\ 2 & \text { if }-1<R<1 \\ R+1 & \text { if } R \geq 1\end{cases}
$$

Thus for any $y \in \mathbb{R}^{l}$

$$
f(0, y) \geq 2 .
$$

On the other side $f(x, y)=0$ for the optimal solution $(x, y)$, that is $x^{j}=$ $0, j \in J$ cannot be the optimal solution.

2) Consider the following sets:

$$
\begin{gathered}
I^{1}=\left\{i \in I: \bar{J}_{i} \neq \emptyset\right\}, \\
I^{2}=\left\{i \in I: \tilde{J}_{i} \neq \emptyset\right\}, \quad I^{3}=I^{1} \bigcap I^{2} .
\end{gathered}
$$

It is clear that $\tilde{J}_{i}=\emptyset$ for any $i \in I^{1} \backslash I^{3}$ and $\bar{J}_{i}=\emptyset$ for any $i \in I^{2} \backslash I^{3}$.

It follows from the definition of the error function that

$$
\begin{aligned}
0=f & (x, y)=\frac{1}{m} \sum_{k=1}^{m} \max \left[0, \max _{i \in I} \min _{j \in J_{i}}\left\{\left\langle x^{j}, a^{k}\right\rangle-y_{j}+1\right\}\right] \\
& +\frac{1}{p} \sum_{t=1}^{p} \max \left[0, \min _{i \in I} \max _{j \in J_{i}}\left\{-\left\langle x^{j}, b^{t}\right\rangle+y_{j}+1\right\}\right] .
\end{aligned}
$$

Since the function $f$ is nonnegative we obtain 


$$
\begin{gathered}
\max _{i \in I} \min _{j \in J_{i}}\left\{\left\langle x^{j}, a\right\rangle-y_{j}+1\right\} \leq 0, \forall a \in A, \\
\min _{i \in I} \max _{j \in J_{i}}\left\{-\left\langle x^{j}, b\right\rangle+y_{j}+1\right\} \leq 0, \forall b \in B .
\end{gathered}
$$

It follows from (17) and (19) that for any $i \in I^{2}$ there exists a point $b \in B$ such that

$$
\max _{j \in J_{i}}\left\{-\left\langle x^{j}, b\right\rangle+y_{j}+1\right\} \leq 0 .
$$

If $i \in I^{3} \subset I^{2}$ then we have

$0 \geq \max _{j \in J_{i}}\left\{-\left\langle x^{j}, b\right\rangle+y_{j}+1\right\}=\max \left\{\max _{j \in \bar{J}_{i}}\left\{-\left\langle x^{j}, b\right\rangle+y_{j}+1\right\}, \max _{i \in \bar{J}_{i}}\left\{y_{j}+1\right\}\right\}$

which means that

$$
\max _{j \in \bar{J}_{i}}\left\{-\left\langle x^{j}, b\right\rangle+y_{j}+1\right\} \leq 0
$$

and

$$
\max _{j \in \tilde{J}_{i}}\left\{y_{j}+1\right\} \leq 0
$$

If $i \in I^{2} \backslash I^{3}$ then from (20) we obtain

$$
0 \geq \max _{j \in J_{i}}\left\{-\left\langle x^{j}, b\right\rangle+y_{j}+1\right\}=\max _{i \in \tilde{J}_{i}}\left\{y_{j}+1\right\}
$$

Thus we get that for all $i \in I^{2}$ the inequality (22) is true. (22) can be rewritten as follows:

$$
\max _{j \in \tilde{J}_{i}} y_{j} \leq-1, \quad \forall i \in I^{2} .
$$

Consequently for any $i \in I^{2}$

$$
\min _{j \in \tilde{J}_{i}}\left\{-y_{j}+1\right\}=-\max _{j \in \tilde{J}_{i}} y_{j}+1 \geq 2 .
$$

It follows from (18) that for any $i \in I$ and $a \in A$

$$
\min _{j \in J_{i}}\left\{\left\langle x^{j}, a\right\rangle-y_{j}+1\right\} \leq 0 .
$$

Then for any $i \in I^{3}$ we have

$$
0 \geq \min _{j \in J_{i}}\left\{\left\langle x^{j}, a\right\rangle-y_{j}+1\right\}=\min \left\{\min _{j \in \bar{J}_{i}}\left\{\left\langle x^{j}, a\right\rangle-y_{j}+1\right\}, \min _{j \in \tilde{J}_{i}}\left\{-y_{j}+1\right\}\right\} .
$$

Taking into account (24) we get that for any $i \in I^{3}$ and $a \in A$

$$
\min _{j \in \bar{J}_{i}}\left\{\left\langle x^{j}, a\right\rangle-y_{j}+1\right\} \leq 0 .
$$

If $i \in I^{2} \backslash I^{3}$ then it follows from (25) that 


$$
\min _{j \in \tilde{J}_{i}}\left\{-y_{j}+1\right\} \leq 0
$$

which contradicts (24). Thus we obtain that $I^{2} \backslash I^{3} \neq \emptyset$ cannot occur, $I^{2} \subset I^{1}$ and $I^{3}=I^{2}$. It is clear that $\vec{J}_{i}=J_{i}$ for any $i \in I^{1} \backslash I^{2}$. Then it follows from (18) that for any $i \in I^{1} \backslash I^{2}$ and $a \in A$

$$
\min _{j \in \bar{J}_{i}}\left\{\left\langle x^{j}, a\right\rangle-y_{j}+1\right\} \leq 0 .
$$

From (26) and (27) we can conclude that for any $i \in I$ and $a \in A$

$$
\min _{j \in \bar{J}_{i}}\left\{\left\langle x^{j}, a\right\rangle-y_{j}+1\right\} \leq 0 \text {. }
$$

It follows from (19) that for any $b \in B$ there exists at least one $i \in I$

$$
\max _{j \in J_{i}}\left\{-\left\langle x^{j}, b\right\rangle+y_{j}+1\right\} \leq 0 \text {. }
$$

Then from expression

$$
\max _{j \in J_{i}}\left\{-\left\langle x^{j}, b\right\rangle+y_{j}+1\right\}=\max \left\{\max _{j \in \bar{J}_{i}}\left\{-\left\langle x^{j}, b\right\rangle+y_{j}+1\right\}, \max _{i \in \tilde{J}_{i}}\left\{y_{j}+1\right\}\right\}
$$

we get that for any $b \in B$ there exists at least one $i \in I$ such that

$$
\max _{j \in \bar{J}_{i}}\left\{-\left\langle x^{j}, b\right\rangle+y_{j}+1\right\} \leq 0 .
$$

Thus it follows from (28) and (29) that the sets $A$ and $B$ are max-min separable with the set of hyperplanes $\left\{x^{j}, y_{j}\right\}, j \in J^{0}$ and a partition $\bar{J}$ of the set $J^{0}$.

Remark 6. In most cases, if a given set of hyperplanes with a particular partition separates the sets $A$ and $B$, then there are other sets of hyperplanes with the same partition which will also separate the sets $A$ and $B$ (see Figure 4). The error function (14) is nonconvex and if the sets $A$ and $B$ are max-min separable, then the global minimum of this function $f\left(x^{*}, y_{*}\right)=0$ and the global minimizer is not unique.

\section{Minimization of the error function}

In this section we discuss an algorithm for minimization of the error function. 


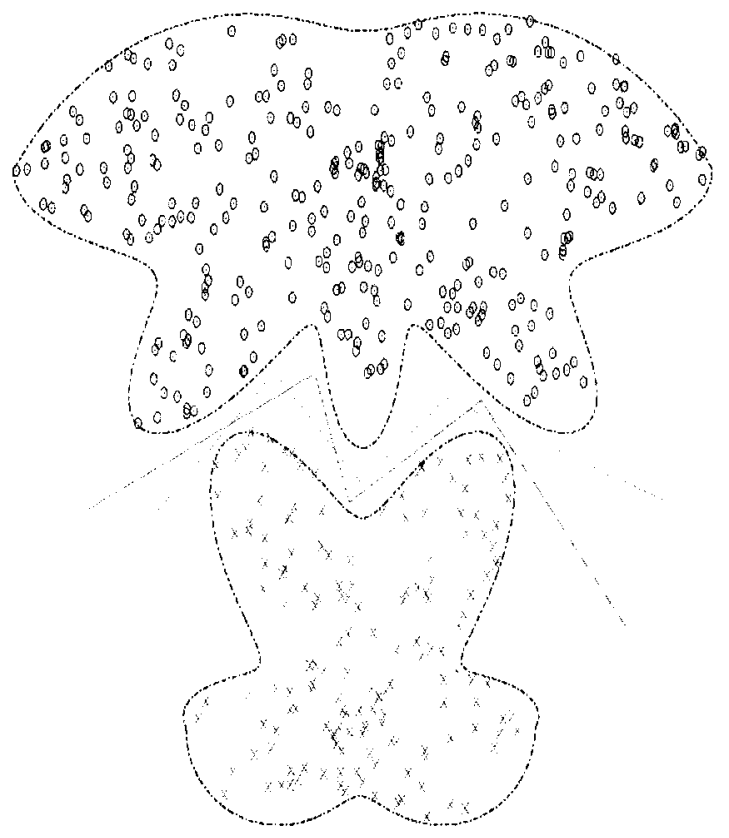

Fig. 4. Max-min separability.

\subsection{Statement of problem}

The problem of the max-min separability is reduced to the following mathematical programming problem:

$$
\text { minimize } f(x, y) \text { subject to }(x, y) \in \mathbb{R}^{(n+1) \times l}
$$

where the objective function $f$ has the following form:

$$
f(x, y)=f_{1}(x, y)+f_{2}(x, y)
$$

and

$$
\begin{aligned}
& f_{1}(x, y)=\frac{1}{m} \sum_{k=1}^{m} \max \left[0, \max _{i \in I} \min _{j \in J_{i}}\left\{\left\langle x^{j}, a^{k}\right\rangle-y_{j}+1\right\}\right], \\
& f_{2}(x, y)=\frac{1}{p} \sum_{t=1}^{p} \max \left[0, \min _{i \in I} \max _{j \in J_{i}}\left\{-\left\langle x^{j}, b^{t}\right\rangle+y_{j}+1\right\}\right] .
\end{aligned}
$$

The problem (30) is a global optimization problem. However, the number of variables in this problem is large and the global optimization methods cannot be directly applied to solve it. Therefore we will discuss algorithms for finding local minima of the function $f$. 
The function $f_{1}$ contains the following $\max -\min$ functions:

$$
\varphi_{1 k}(x, y)=\max _{i \in I} \min _{j \in J_{i}}\left\{\left\langle x^{j}, a^{k}\right\rangle-y_{j}+1\right\}, \quad k=1, \ldots, m
$$

and the function $f_{2}$ contains the following min-max functions:

$$
\varphi_{2 t}(x, y)=\min _{i \in I} \max _{j \in J_{i}}\left\{-\left\langle x^{j}, b^{t}\right\rangle+y_{j}+1\right\}, \quad t=1, \ldots, p .
$$

\subsection{Differential properties of the objective function}

Both functions $f_{1}$ and $f_{2}$ are nonsmooth, nonconvex piecewise linear. These functions contain some max-min-type functions. The functions $f_{1}$ and $f_{2}$ and consequently, the function $f$ are locally Lipschitz continuous. We will recall some definitions from nonsmooth analysis.

We consider a locally Lipschitz function $\varphi$ defined on $\mathbb{R}^{n}$. This function is differentiable almost everywhere and one can define for it a Clarke subdifferential (see [Cla83]), by

$$
\begin{aligned}
& \partial \varphi(x) \\
= & \operatorname{co}\left\{v \in \mathbb{R}^{n}: \exists\left(x^{k} \in D(\varphi), x^{k} \longrightarrow x, k \longrightarrow+\infty\right): v=\lim _{k \longrightarrow+\infty} \nabla \varphi\left(x^{k}\right)\right\},
\end{aligned}
$$

here $D(\varphi)$ denotes the set where $\varphi$ is differentiable, co denotes the convex hull of a set.

The function $\varphi$ is differentiable at the point $x \in \mathbb{R}^{n}$ with respect to the direction $g \in \mathbb{R}^{n}$ if the limit

$$
\varphi^{\prime}(x, g)=\lim _{\alpha \rightarrow+0} \frac{\varphi(x+\alpha g)-\varphi(x)}{\alpha}
$$

exists. The number $\varphi^{\prime}(x, g)$ is said to be the derivative of the function $\varphi$ with respect to the direction $g \in \mathbb{R}^{n}$ at the point $x$.

The Clarke upper derivative $\varphi^{0}(x, g)$ of the function $\varphi$ at the point $x$ with respect to the direction $g \in \mathbb{R}^{n}$ is defined as follows:

$$
\varphi^{0}(x, g)=\limsup _{\alpha \rightarrow+0, y \rightarrow x} \frac{\varphi(y+\alpha g)-\varphi(y)}{\alpha} .
$$

The following is true (see [Cla83])

$$
\varphi^{0}(x, g)=\max \{\langle v, g\rangle: v \in \partial \varphi(x)\} .
$$

It should be noted that the Clarke upper derivative always exists for locally Lipschitz continuous functions. The function $\varphi$ is said to be Clarke regular at the point $x \in \mathbb{R}^{n}$ if

$$
\varphi^{\prime}(x, g)=\varphi^{0}(x, g)
$$


for all $g \in \mathbb{R}^{n}$. For Clarke regular functions there exists a calculus (see [Cla83, DR95]). However in general for non-regular functions such a calculus does not exist.

The function $\varphi$ is called semismooth at $x \in \mathbb{R}^{n}$, if it is locally Lipschitz continuous at $x$ and for every $g \in \mathbb{R}^{n}$, the limit

$$
\lim _{v \in \partial \varphi\left(x+t g^{\prime}\right), g^{\prime} \rightarrow g, t \rightarrow+0}\langle v, g\rangle
$$

exists (see [Mif77]).

Let us return to the objective function $f$ of problem (30). Since this function is locally Lipschitz continuous it is Clarke subdifferentiable.

Proposition 7. The function $f$ is semismooth.

Proof. The sum, the maximum and the minimum of semismooth functions are semismooth (see [Mif77]). A linear function, as a smooth function, is semismooth. Thus the function $f$ which is the sum of functions represented as the maximum of 0 and max-min of linear functions, is semismooth.

The properties of max-min type functions were studied, for example, in [DDM02, Pol97]. Max-min-type functions in general are not Clarke regular.

Example 1. Consider the function

$$
\varphi(x)=\max \left\{\min \left\{3 x_{1}+x_{2}, 2 x_{1}+3 x_{2}\right\}, \min \left\{x_{1}+2 x_{2}, 4 x_{1}+4 x_{2}\right\}\right\} .
$$

The Clarke subdifferential of this function at the point $x=(0,0)$ is

$$
\partial \varphi(x)=\operatorname{co}\{(3,1),(2,3),(1,2),(4,4)\}
$$

Then the Clarke upper derivative $\varphi^{0}\left(x, g^{0}\right)$ of the function $\varphi$ at the point $x=(0,0)$ with respect to the direction $g^{0}=(0,1)$ is

$$
\varphi^{0}\left(x, g^{0}\right)=\max \left\{\left\langle v, g^{0}\right\rangle: v \in \partial \varphi(x)\right\}=4 .
$$

However, the directional derivative of this function with respect to the direction $g^{0}=(0,1)$ is $\varphi^{\prime}(x, g)=2$ that is $\varphi^{\prime}\left(x, g^{0}\right)<\varphi^{0}\left(x, g^{0}\right)$. Thus the function $\varphi$ is not Clarke regular.

Since the function $f$ contains max-min of linear functions this function is not Clarke regular apart from linear separability. Therefore, subgradients of the function $f$ cannot be calculated using subgradients of the involved maxmin-type functions. We can conclude that the calculation of the subgradients of the function $f$ is a very difficult task and therefore the application of methods of nonsmooth optimization requiring a subgradient evaluation at each iteration, including bundle method and its variations([HL93, Kiw85, MN92]), cannot be effective. 
In the paper [KP98] optimization problems with twice continuously differentiable objective functions and max-min constraints were considered and these problems were converted to problems with smooth objective and constraint functions. However, this approach cannot be applied to the problem (30), because the function $f$ contains not only max-min-type functions but also min-max-type functions.

Since the evaluation of subgradients of the function $f$ is difficult, direct search methods of optimization seem to be the best option for solving problem (30). Among such methods we mention here two widely used methods: Powell's method (see [Pow02]) which is based on a quadratic approximation of the objective function and Nelder-Mead's simplex method [NM65]. As was mentioned in [Pow02] Powell's method performs well when the number of variables is less than 20. For the simplex method this number is even smaller. Moreover, both methods are effective when the objective function is smooth. However, in the max-min separability problem the number of variables is $n_{v}=(n+1) \times l$ where $n$ is the dimension of the sets $A$ and $B$ (ranging from 5 to thousands in real world datasets), and $l$ is the number of separating hyperplanes. In many cases the number $n_{v}$ is greater than 20. Furthermore, the objective function in this problem is a quite complicated nonsmooth function.

In this paper we use the discrete gradient method to solve the problem (30). The description of this method can be found in [Bag99a, Bag99b] (see, also, [Bag02]). The discrete gradient method can be considered as a version of the bundle method ([HL93, Kiw85, MN92]), where subgradients of the objective function are replaced by its discrete gradients.

The discrete gradient method uses only values of the objective function. It should be noted that the calculation of the objective function in the problem (30) can be expensive. We will show that the use of the discrete gradient method allow to significantly reduce the number of objective function evaluations.

\subsection{Discrete gradient method}

In this subsection we will briefly describe the discrete gradient method. We start with the definition of the discrete gradient.

\section{Definition of the discrete gradient}

Let $f$ be a locally Lipschitz continuous function defined on $\mathbb{R}^{n}$. Let

$$
\begin{aligned}
S_{1} & =\left\{g \in \mathbb{R}^{n}:\|g\|=1\right\}, \\
G & =\left\{e \in \mathbb{R}^{n}: e=\left(e_{1}, \ldots, e_{n}\right),\left|e_{j}\right|=1, j=1, \ldots, n\right\}, \\
P & =\left\{z(\lambda): z(\lambda) \in \mathbb{R}^{1}, z(\lambda)>0, \lambda>0, \lambda^{-1} z(\lambda) \rightarrow 0, \lambda \rightarrow 0\right\}, \\
I(g, \alpha) & =\left\{i \in\{1, \ldots, n\}:\left|g_{i}\right| \geq \alpha\right\},
\end{aligned}
$$

where $\alpha \in\left(0, n^{-1 / 2}\right]$ is a fixed number. 
Here $S_{1}$ is the unit sphere, $G$ is the set of vertices of the unit hypercube in $\mathbb{R}^{n}$ and $P$ is the set of univariate positive infinitesimal functions.

We define operators $H_{i}^{j}: \mathbb{R}^{n} \rightarrow \mathbb{R}^{n}$ for $i=1, \ldots, n, j=0, \ldots, n$ by the formula

$$
H_{i}^{j} g= \begin{cases}\left(g_{1}, \ldots, g_{j}, 0, \ldots, 0\right) & \text { if } j<i \\ \left(g_{1}, \ldots, g_{i-1}, 0, g_{i+1}, \ldots, g_{j}, 0, \ldots, 0\right) & \text { if } j \geq i\end{cases}
$$

We can see that

$$
H_{i}^{j} g-H_{i}^{j-1} g=\left\{\begin{array}{cl}
\left(0, \ldots, 0, g_{j}, 0, \ldots, 0\right) & \text { if } j=1, \ldots, n, j \neq i \\
0 & \text { if } j=i .
\end{array}\right.
$$

Let $e(\beta)=\left(\beta e_{1}, \beta^{2} e_{2}, \ldots, \beta^{n} e_{n}\right)$, where $\beta \in(0,1]$. For $x \in \mathbb{R}^{n}$ we consider vectors

$$
x_{i}^{j} \equiv x_{i}^{j}(g, e, z, \lambda, \beta)=x+\lambda g-z(\lambda) H_{i}^{j} e(\beta),
$$

where $g \in S_{1}, e \in G, i \in I(g, \alpha), z \in P, \lambda>0, j=0, \ldots, n, j \neq i$.

It follows from (34) that

$$
x_{i}^{j-1}-x_{i}^{j}=\left\{\begin{array}{cl}
\left(0, \ldots, 0, z(\lambda) e_{j}(\beta), 0, \ldots, 0\right) & \text { if } j=1, \ldots, n, j \neq i \\
0 & \text { if } j=i .
\end{array}\right.
$$

It is clear that $H_{i}^{0} g=0$ and $x_{i}^{0}(g, e, z, \lambda, \beta)=x+\lambda g$ for all $i \in I(g, \alpha)$.

Definition 4. (see [BG95]) The discrete gradient of the function $f$ at the point $x \in \mathbb{R}^{n}$ is the vector $\Gamma^{i}(x, g, e, z, \lambda, \beta)=\left(\Gamma_{1}^{i}, \ldots, \Gamma_{n}^{i}\right) \in \mathbb{R}^{n}, g \in S_{1}, i \in$ $I(g, \alpha)$, with the following coordinates:

$$
\begin{gathered}
\Gamma_{j}^{i}=\left[z(\lambda) e_{j}(\beta)\right]^{-1}\left[f\left(x_{i}^{j-1}(g, e, z, \lambda, \beta)\right)-f\left(x_{i}^{j}(g, e, z, \lambda, \beta)\right)\right], \\
j=1, \ldots, n, j \neq i \\
\Gamma_{i}^{i}=\left(\lambda g_{i}\right)^{-1}\left[f\left(x_{i}^{n}(g, e, z, \lambda, \beta)\right)-f(x)-\sum_{j=1, j \neq i}^{n} \Gamma_{j}^{i}\left(\lambda g_{j}-z(\lambda) e_{j}(\beta)\right)\right] .
\end{gathered}
$$

A more detailed description of the discrete gradient and examples can be found in [Bag99b].

Remark 7. It follows from Definition 4 that for the calculation of the discrete gradient $\Gamma^{i}(x, g, e, z, \lambda, \beta), i \in I(g, \alpha)$ we define a sequence of points

$$
x_{i}^{0}, \ldots, x_{i}^{i-1}, x_{i}^{i+1}, \ldots, x_{i}^{n} .
$$

For the calculation of the discrete gradient it is sufficient to evaluate the function $f$ at each point of this sequence. 
Remark 8. The discrete gradient is defined with respect to a given direction $g \in S_{1}$. We can see that for the calculation of one discrete gradient we have to calculate $(n+1)$ values of the function $f$ : at the point $x$ and at the points $x_{i}^{j}(g, e, z, \lambda, \beta), j=0, \ldots, n, j \neq i$. For the calculation of the next discrete gradient at the same point with respect to any other direction $g^{1} \in S_{1}$ we have to calculate this function $n$ times, because we have already calculated $f$ at the point $x$.

\section{Calculation of the discrete gradients of the objective function (30)}

Now let us return to the objective function $f$ of the problem (30). This function depends on $(n+1) l$ variables where $l$ is the number of hyperplanes. The function $f_{1}$ contains max-min functions $\varphi_{1 k}$

$$
\varphi_{1 k}(x, y)=\max _{i \in I} \min _{j \in J_{i}} \psi_{1 j k}(x, y), k=1, \ldots, m
$$

where

$$
\psi_{1 j k}(x, y)=\left\langle x^{j}, a^{k}\right\rangle-y_{j}+1, j \in J_{i}, i \in I .
$$

We can see that for every $k=1, \ldots, m$, each pair of variables $\left\{x^{j}, y_{j}\right\}$ appears in only one function $\psi_{1 j k}$.

For a given $i=1, \ldots,(n+1) l$ we set

$$
q_{i}=\left\lfloor\frac{i-1}{n+1}\right\rfloor+1, \quad d_{i}=i-\left(q_{i}-1\right)(n+1)
$$

where $\lfloor u\rfloor$ stands for the floor of a number $u$. We define by $X$ the vector of all variables $\left\{x^{j}, y_{j}\right\}, j=1, \ldots, l$ :

$$
X=\left(X_{1}, X_{2}, \ldots, X_{(n+1) l}\right)
$$

where

$$
X_{i}= \begin{cases}x_{d_{i}}^{q_{i}} & \text { if } 1 \leq d_{i} \leq n \\ y_{q_{i}} & \text { if } d_{i}=n+1\end{cases}
$$

We use the vector of variables $X$ to define a sequence

$$
X_{t}^{0}, \ldots, X_{t}^{t-1}, X_{t}^{t+1}, \ldots, X_{t}^{(n+1) l}, t \in I(g, \alpha), g \in \mathbb{R}^{(n+1) l}
$$

as in Remark 7. It follows from (36) that the points $X_{t}^{i-1}$ and $X_{t}^{i}$ differ by one coordinate only. This coordinate appears in only one linear function $\psi_{1 q_{i} k}$. It follows from the definition of the operator $H_{i}^{j}$ that $X_{t}^{t}=X_{t}^{t-1}$ and thus this observation is also true for $X_{t}^{t+1}$. Then we get

$$
\psi_{1 j k}\left(X_{t}^{i}\right)=\psi_{1 j k}\left(X_{t}^{i-1}\right) \forall j \neq q_{i}
$$

Moreover the function $\psi_{1 q_{i} k}$ can be calculated at the point $X_{t}^{i}$ using the value of this function at the point $X_{t}^{i-1}, i \geq 1$ : 


$$
\psi_{1 q_{i} k}\left(X_{t}^{i}\right)= \begin{cases}\psi_{1 q_{i} k}\left(X_{t}^{i-1}\right)-z(\lambda) a_{d_{i}}^{k} e_{i}(\beta) & \text { if } 1 \leq d_{i} \leq n \\ \psi_{1 q_{i} k}\left(X_{t}^{i-1}\right)+z(\lambda) e_{i}(\beta) & \text { if } d_{i}=n+1\end{cases}
$$

In order to calculate the function $f_{1}$ at the point $X_{t}^{i}, i \geq 1$ first we have to calculate the values of the functions $\psi_{1 q_{i} k}$ for all $a^{k} \in A, k=1, \ldots, m$ using (37). Then we update $f_{1}$ using these values and the values of all other linear functions at the point $X_{t}^{i-1}$ according to (31). Thus we have to apply a full calculation of the function $f_{1}$ using the formula(31) only at the point $X_{t}^{0}=X+\lambda g$.

Since the function $f_{2}$ has a similar structure as $f_{1}$ we can calculate it in the same manner using a formula similar to (37).

Thus for the calculation of each discrete gradient we have to apply a full calculation of the objective function $f$ only at the point $X_{t}^{0}=X+\lambda g$ and this function can be updated at the points $X_{t}^{i}, i \geq 1$ using a simplified scheme.

We can conclude that for the calculation of the discrete gradient at a point $X$ with respect to the direction $g^{0} \in S_{1}$ we calculate the function $f$ at two points: $X$ and $X_{t}^{0}=X+\lambda g^{0}$. For the calculation of another discrete gradient at the same point $X$ with respect to any other direction $g^{1} \in S_{1}$ we calculate the function $f$ only at the point: $X+\lambda g^{1}$.

Since the number of variables $(n+1) l$ in the problem $(30)$ can be large this algorithm allows to significantly reduce the number of objective function evaluations during the calculation of a discrete gradient.

On the other hand the function $f_{1}$ contains max-min-type functions and their computation can be simplified using an algorithm proposed in [Evt72]. The function $f_{2}$ contains min-max-type functions and a similar algorithm can be used for their calculation.

Results of numerical experiments show that the use of these algorithms allows one to significantly accelerate the computation of the objective function $f$ and its discrete gradients.

\section{Discrete gradient method}

We consider the following unconstrained minimization problem:

$$
\text { minimize } \varphi(x) \text { subject to } x \in \mathbb{R}^{n}
$$

where the function $\varphi$ is assumed to be semismooth. We consider the following algorithm for solving this problem. An important step in this algorithm is the calculation of a descent direction of the objective function $\varphi$. So first, we describe an algorithm for the computation of this descent direction.

Let $z \in P, \lambda>0, \beta \in(0,1]$, the number $c \in(0,1)$ and a small enough number $\delta>0$ be given.

Algorithm 1. An algorithm for the computation of the descent direction. 
Step 1. Choose any $g^{1} \in S_{1}, e \in G, i \in I\left(g^{1}, \alpha\right)$ and compute a discrete gradient $v^{1}=\Gamma^{i}\left(x, g^{1}, e, z, \lambda, \beta\right)$. Set $\bar{D}_{1}(x)=\left\{v^{1}\right\}$ and $k=1$.

Step 2. Calculate the vector $\left\|w^{k}\right\|=\min \left\{\|w\|: w \in \bar{D}_{k}(x)\right\}$. If

$$
\left\|w^{k}\right\| \leq \delta
$$

then stop. Otherwise go to Step 3.

Step 3. Calculate the search direction by $g^{k+1}=-\left\|w^{k}\right\|^{-1} w^{k}$.

Step 4. If

$$
\varphi\left(x+\lambda g^{k+1}\right)-\varphi(x) \leq-c \lambda\left\|w^{k}\right\|
$$

then stop. Otherwise go to Step 5.

Step 5. Calculate a discrete gradient

$$
v^{k+1}=\Gamma^{i}\left(x, g^{k+1}, e, z, \lambda, \beta\right), i \in I\left(g^{k+1}, \alpha\right),
$$

construct the set $\bar{D}_{k+1}(x)=\operatorname{co}\left\{\bar{D}_{k}(x) \bigcup\left\{v^{k+1}\right\}\right\}$, set $k=k+1$ and go to Step 2.

Algorithm 1 contains some steps which deserve some explanations. In Step 1 we calculate the first discrete gradient. The distance between the convex hull of all calculated discrete gradients and the origin is calculated in Step 2. If this distance is less than the tolerance $\delta>0$ then we accept the point $x$ as an approximate stationary point (Step 2), otherwise we calculate another search direction in Step 3. In Step 4 we check whether this direction is a descent direction. If it is we stop and the descent direction has been calculated, otherwise we calculate another discrete gradient with respect to this direction in Step 5 and add it to the set $\bar{D}_{k}$.

It is proved that Algorithm 1 is terminating (see [Bag99a, Bag99b]).

Let numbers $c_{1} \in(0,1), c_{2} \in\left(0, c_{1}\right]$ be given.

\section{Algorithm 2. Discrete gradient method}

Step 1. Choose any starting point $x^{0} \in \mathbb{R}^{n}$ and set $k=0$.

Step 2. Set $s=0$ and $x_{s}^{k}=x^{k}$.

Step 3. Apply Algorithm 1 for the calculation of the descent direction at $x=x_{s}^{k}, \delta=\delta_{k}, z=z_{k}, \lambda=\lambda_{k}, \beta=\beta_{k}, c=c_{1}$. This algorithm terminates after a finite number of iterations $m>0$. As a result we get the set $D_{m}\left(x_{s}^{k}\right)$ and an element $v_{s}^{k}$ such that

$$
\left\|v_{s}^{k}\right\|=\min \left\{\|v\|: v \in \bar{D}_{m}\left(x_{s}^{k}\right)\right\} .
$$

Furthermore either $\left\|v_{s}^{k}\right\| \leq \delta_{k}$ or for the search direction $g_{s}^{k}=-\left\|v_{s}^{k}\right\|^{-1} v_{s}^{k}$ 


$$
\varphi\left(x_{s}^{k}+\lambda_{k} g_{s}^{k}\right)-\varphi\left(x_{s}^{k}\right) \leq-c_{1} \lambda_{k}\left\|v_{s}^{k}\right\|
$$

Step 4. If

$$
\left\|v_{s}^{k}\right\| \leq \delta_{k}
$$

then set $x^{k+1}=x_{s}^{k}, k=k+1$ and go to Step 2. Otherwise go to Step 5 .

Step 5. Construct the following iteration $x_{s+1}^{k}=x_{s}^{k}+\sigma_{s} g_{s}^{k}$, where $\sigma_{s}$ is defined as follows

$$
\sigma_{s}=\arg \max \left\{\sigma \geq 0: \varphi\left(x_{s}^{k}+\sigma g_{s}^{k}\right)-\varphi\left(x_{s}^{k}\right) \leq-c_{2} \sigma\left\|v_{s}^{k}\right\|\right\} .
$$

Step 6. Set $s=s+1$ and go to Step 3 .

For the point $x^{0} \in \mathbb{R}^{n}$ we consider the set $M\left(x^{0}\right)=\left\{x \in \mathbb{R}^{n}: \varphi(x) \leq\right.$ $\left.\varphi\left(x^{0}\right)\right\}$.

Theorem 1. Assume that the set $M\left(x^{0}\right)$ is bounded for starting points $x^{0} \in$ $\mathbb{R}^{n}$. Then every accumulation point of $\left\{x^{k}\right\}$ belongs to the set $X^{0}=\{x \in$ $\left.\mathbb{R}^{n}: 0 \in \partial \varphi(x)\right\}$.

Since the objective function in problem (30) is semismooth the discrete gradient method can be applied to solve it. Discrete gradients in Step 5 of Algorithm 1 can be calculated using the simplified scheme described above.

\section{Results of numerical experiments}

We applied the max-min separation to solve supervised data classification problems on some real-world datasets. In this section we present results of numerical experiments. Our algorithm has been implemented in Lahey Fortran 95 on a Pentium $41.7 \mathrm{GHz}$.

\subsection{Supervised data classification via max-min separability}

We are given a dataset $A$ containing a finite number of points in $\mathbb{R}^{n}$. This dataset contains $d$ disjoint subsets $A_{1}, \ldots, A_{d}$ where $A_{i}$ represents a training set for the class $i$. The aim of supervised data classification is to establish rules for the classification of some new observations using these training subsets of the classes. This problem is reduced to $d$ set separation problems.

Each of these problems consists in separating one class from the rest of the dataset. To separate the class $i$ from all others, we separate sets $A_{i}$ and $\bigcup_{j \neq i} A_{j}$, with a piecewise linear function by solving problem (30).

One of the important question in supervised data classification is the estimation of performance measure. Different performance measures are discussed in [Tho02]. When the dataset contains two classes the classification problem 
can be reduced to only one separation problem, therefore the classification rules are straightforward. We consider that the separation function obtained from the training set, separates the two classes.

When the dataset contains more than two classes we have more than one separation function. In our case for each class $i$ of the dataset $A$ we have one piecewise linear function $\varphi_{i}$ separating the training set $A_{i}$ from all other training points $\bigcup_{j \neq i} A_{j}$. We approximate the training set $A_{i}$ using the following set

$$
\bar{A}_{i}=\left\{a \in \mathbb{R}^{n}: \varphi_{i}(a)<0\right\} .
$$

Thus we get the sets $\bar{A}_{1}, \ldots, \bar{A}_{d}$ which approximate the training sets $A_{1}, \ldots, A_{d}$, respectively. Then for each $i \in\{1, \ldots, d\}$ we can consider the following two sets:

$$
A_{i}^{0}=\bar{A}_{i}, \quad \bar{A}_{i}^{0}=\bigcup_{j=1, j \neq i}^{d} \bar{A}_{j}
$$

These two sets define the following four sets (see Figure 5):

1. $A_{i}^{0} \bigcap\left(\mathbb{R}^{n} \backslash \bar{A}_{i}^{0}\right)$

2. $\left(\mathbb{R}^{n} \backslash A_{i}^{0}\right) \cap \bar{A}_{i}^{0}$

3. $A_{i}^{0} \cap \bar{A}_{i}^{0}$

4. $\left(\mathbb{R}^{n} \backslash A_{i}^{0}\right) \bigcap\left(\mathbb{R}^{n} \backslash \bar{A}_{i}^{0}\right)$

If a new observation $a$ belongs to the first set we classify it in class $i$, if it belongs to the second set we classify it not to be in class $i$. If this point belongs to the third or fourth set in this case if $\varphi_{i}(a)<\min _{j=1, \ldots, d, j \neq i} \varphi_{j}(a)$ then we classify it in class $i$, otherwise we classify it not to be in class $i$.

In order to evaluate the classification algorithm we use two performance measures. First we present the average accuracy $\left(a_{2 c}\right.$ in Tables 3 and 4$)$ for well-classified points in two classes classification (when one particular class is separated from all others) and the multi-class classification accuracy ( $a_{m c}$ in Tables 3 and 4) as described above. First accuracy is an indication of separation quality and the second one is an indication of multi-class classification quality.

\subsection{Results on small and middle size datasets}

In this subsection we present results of numerical experiments with some small and middle size datasets in order to demonstrate the separation ability of the proposed algorithm. The datasets used are the Wisconsin Breast Cancer Diagnosis (WBCD), the Wisconsin Breast Cancer Prognosis (WBCP), the Cleveland Heart Disease (Heart), the Pima Indians Diabetes (Diabetes), the BUPA Liver Disorders (Liver), the United States Congressional Voting Records (Votes) and the Ionosphere. All datasets contain 2 classes. The description of these datasets can be found in [MA92].

We take entire datasets and check their polyhedral or max-min separability considering various number of hyperplanes. Results of numerical experiments 


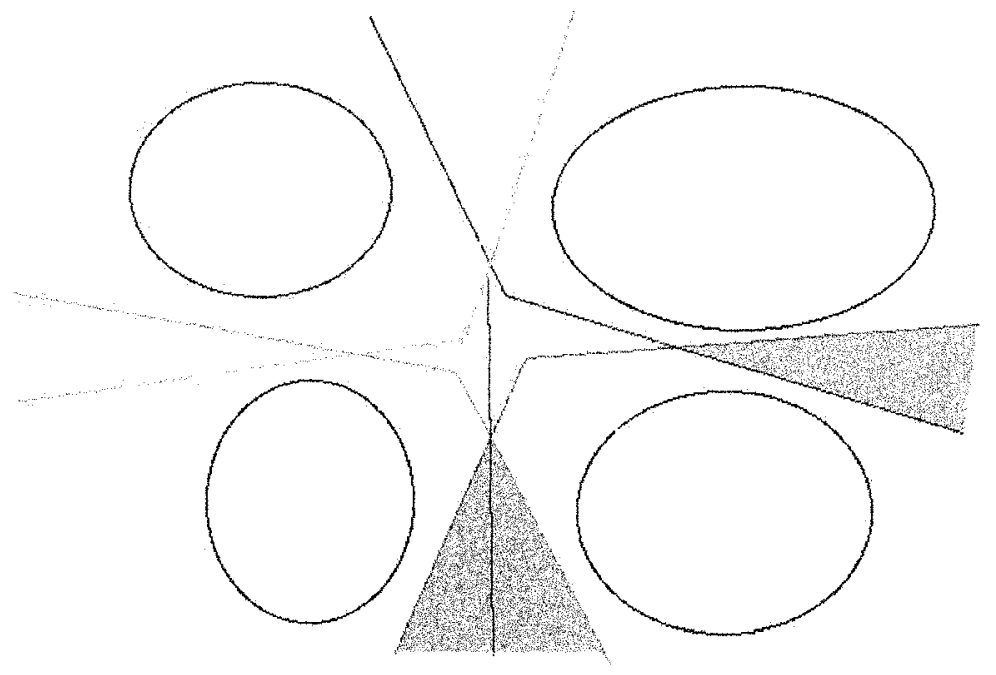

Fig. 5. Multi-class classification by a max-min separation

are presented in Table 1. We use the following notation: $m$ - is the number of instances in the first class, $p$ - is the number of instances in the second class, $n$ - number of attributes, $h$ number of hyperplanes used for polyhedral separability, $r$ is the cardinality of the set $I$ and $j$ is the cardinality of the sets $J_{i}, i \in I$ in the max-min separability. The sets $J_{i}$ contain the same number of indices for all $i \in I$. In our experiments we restrict $r$ to 15 and $j$ to 5 . The accuracy is defined as the ratio between the number of well-classified points of both $A$ and $B$ and the total number of points in the dataset.

Table 1. Results of numerical experiments with small and middle size datasets

\begin{tabular}{ccccccc}
\hline Database & $m / p / n$ & Linear & $\begin{array}{c}\text { Polyhedral } \\
\text { accuracy }\end{array}$ & \multicolumn{2}{c}{ Max-min } \\
& & & \multicolumn{1}{c}{$h$ accuracy } \\
\hline WBCD & $239 / 444 / 9$ & 97.36 & 7 & 98.98 & $5 \times 2$ & 100 \\
Heart & $137 / 160 / 13$ & 84.19 & 10 & 100 & $2 \times 5$ & 100 \\
Ionosphere & $126 / 225 / 34$ & 93.73 & 4 & 97.44 & $2 \times 2$ & 100 \\
Votes & $168 / 267 / 16$ & 96.80 & 5 & 100 & $2 \times 3$ & 100 \\
WBCP & $46 / 148 / 32$ & 76.80 & 4 & 100 & $3 \times 2$ & 100 \\
Diabetes & $268 / 500 / 8$ & 76.95 & 12 & 80.60 & $15 \times 2$ & 90.10 \\
Liver & $145 / 200 / 6$ & 68.41 & 12 & 74.20 & $6 \times 5$ & 89.86 \\
\hline
\end{tabular}

From the results presented in Table 1 we can conclude that in none of the datasets classes are linearly separable. Classes in heart, votes and WBCP are polyhedrally separable and in WBCD they are "almost" polyhedrally sep- 
arable. We considered different values for $h$ in diabetes and liver datasets and present best results. These results show that classes in these datasets are unlikely to be polyhedrally separable. Classes in WBCD, heart, ionosphere, votes and WBCP are max-min separable with a presented number of hyperplanes whereas classes in diabetes and liver datasets are likely to be max-min separable with quite large number of hyperplanes. On the other side results for these datasets show that the use of max-min separability allows one to achieve significantly better separation.

\subsection{Results on larger datasets}

\section{Datasets}

The datasets used are the Shuttle control, the Letter recognition, the Landsat satellite image, the Pen-based recognition of handwritten and the Page blocks classification databases. Table 2 presents some characteristics of these databases. More detailed information can be found in [MA92]. It should be noted that all attributes in these datasets are continuous.

Table 2. Large datasets

\begin{tabular}{cccc}
\hline Database & (train,test) & $\begin{array}{c}\text { No. of } \\
\text { attributes classes }\end{array}$ & $\begin{array}{c}\text { No. of } \\
\text { classe }\end{array}$ \\
\hline $\begin{array}{c}\text { Shuttle control } \\
\text { Letter recognition }\end{array}$ & $(43500,14500)$ & 9 & 7 \\
Landsat satellite image & $(44000,5000)$ & 16 & 26 \\
Pen-based recognition of & & 36 & 6 \\
handwritten & $(7494,3498)$ & 16 & 10 \\
Page blocks & $(4000,1473)$ & 10 & 5 \\
\hline
\end{tabular}

\section{Results and discussion}

We took $X^{0}=0 \in \mathbb{R}^{(n+1) l}$ as a starting point for solving each separation problem (30). At each iteration of the discrete gradient method the line search is carried out by approximation of the objective function using univariate piecewise linear function (see [Bag99a]). In each separation problem (30) all $J_{i}, i \in I$ have the same cardinality.

Results of numerical experiments are presented in Tables 3 and 4 . In these tables fct eval, $D G$ eval and $C P U$ time show respectively the average number of objective function evaluations, discrete gradient evaluations and CPU time required to solve an optimization problem. CPU time is presented in seconds.

From the results presented in these tables we can see that the use of the max-min separability algorithm allows to achieve a high classification 
accuracy for both training and test phases. Results on training sets show that this algorithm provides a high quality of separation between two sets. In our experiments we used only large-scale datasets. Results on these datasets show that a few hyperplanes are sufficient to separate efficiently sets with large numbers of points. Since we use a derivative-free method to solve problem (30) the number of objective function evaluations is a significant characteristic for estimation of the complexity of the max-min separability algorithm. Results presented in Tables 3 and 4 confirm that the proposed algorithm is effective for solving classification problems on large-scale databases.

Table 3. Results of numerical experiments with Shuttle control, Letter recognition and Landsat satellite image datasets

\begin{tabular}{|c|c|c|c|c|c|c|c|c|}
\hline & & Trai & ning & $\mathrm{Te}$ & est & & & \\
\hline$|I|$ & $\left|J_{i}\right|$ & $a_{2 c}$ & $a_{m c}$ & $a_{2 \mathrm{c}}$ & $a_{m c}$ & fct eva & DG eval & CPU time \\
\hline & & & Shuttle & contr & rol dat & aset & & \\
\hline 1 & 1 & 94.63 & 87.84 & 94.66 & 87.86 & 265 & 268 & $54 . \overline{44}$ \\
\hline 2 & 1 & 97.26 & 97.58 & 97.08 & 97.49 & 396 & 399 & 145.12 \\
\hline 3 & 1 & 97.04 & 99.36 & 96.87 & 99.21 & 379 & 379 & 211.23 \\
\hline 4 & 1 & 97.35 & 99.50 & 97.19 & 99.35 & 402 & 405 & 310.54 \\
\hline 2 & 2 & 99.86 & 99.57 & 99.86 & 99.39 & 391 & 394 & 281.92 \\
\hline 3 & 2 & 99.48 & 99.92 & 99.43 & 99.86 & 636 & 639 & 825.99 \\
\hline 4 & 2 & 99.84 & 99.76 & 99.82 & 99.70 & 447 & 450 & 810.58 \\
\hline & & & tter re & cognit & tion $\mathrm{d}$ & ataset & & \\
\hline 1 & 1 & 92.51 & 66.89 & 92.32 & 66.00 & 280 & 284 & 17.57 \\
\hline 2 & 1 & 96.83 & 79.86 & 95.24 & 79.36 & 568 & 572 & 60.98 \\
\hline 3 & 1 & 98.34 & 85.73 & 95.94 & 84.82 & 573 & 575 & 93.72 \\
\hline 4 & 1 & 99.08 & 89.32 & 96.36 & 86.86 & 665 & 667 & 158.29 \\
\hline 2 & 2 & 98.12 & 86.89 & 96.20 & 84.56 & 683 & 686 & 143.07 \\
\hline 3 & 2 & 98.97 & 91.46 & 96.32 & 89.12 & 634 & 635 & 366.16 \\
\hline 3 & 3 & 99.52 & 93.73 & 96.16 & 90.32 & 511 & 511 & 436.37 \\
\hline & & Land & sat sat & ellite & image & datase & & \\
\hline 1 & 1 & 93.12 & $86.00 !$ & 91.30 & 83.45 & 298 & 301 & 4.62 \\
\hline 2 & 1 & 96.73 & 88.129 & 94.40 & 85.65 & 549 & 552 & 19.12 \\
\hline 3 & 1 & 97.54 & $89.80 \mathrm{~g}$ & 94.80 & 87.00 & 618 & 621 & 37.37 \\
\hline 4 & 1 & 97.81 & $91.14 \mathrm{~g}$ & 94.35 & 87.45 & 656 & 659 & 61.64 \\
\hline 2 & 2 & 97.56 & 90.859 & 94.25 & 87.10 & 606 & 609 & 48.83 \\
\hline 3 & 2 & 98.02 & 90.989 & 94.60 & 86.70 & 712 & 715 & 116.86 \\
\hline 4 & 2 & 98.47 & 93.339 & 94.80 & 86.70 & 533 & 536 & 137.07 \\
\hline
\end{tabular}

\section{Conclusions and further work}

In this paper we have developed the concept of the max-min separability. If finite point sets $A$ and $B$ are disjoint then they can be separated by a certain 
Table 4. Results of numerical experiments with Pen-based recognition of handwritten and Page blocks datasets

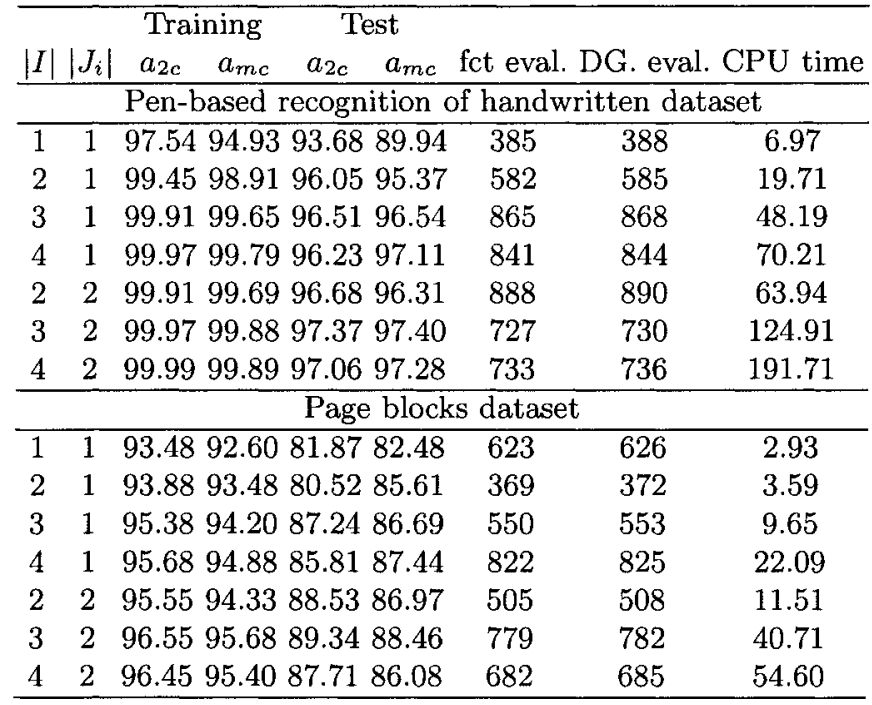

piecewise linear function presented as a max-min of linear functions. We have proposed an algorithm to find this piecewise linear function by minimizing an error function.

This algorithm has been applied to solve data classification problems in some large-scale datasets. Results from numerical experiment show the effectiveness of this algorithm.

However the number of hyperplanes needed to separate the two sets has to be known. In further research some methods to find automatically this number will be introduced. Problem (30) is a global optimization problem on which we use a local optimization method. Therefore it is very crucial to find a good initial point in order to reduce computational cost and to improve the solution. These questions are the subject of our further research.

\section{Acknowledgements}

This research was supported by the Australian Research Council.

\section{References}

[AG02] Astorino, A., Gaudioso, M.: Polyhedral separability through successive LP. Journal of Optimization Theory and Applications, 112, 265-293 (2002) 
[BG95] Bagirov, A.M., Gasanov, A.A.: A method of approximating a quasidifferential. Russian Journal of Computational Mathematics and Mathematical Physics, 35, 403-409 (1995)

[Bag99a] Bagirov, A.M.: Derivative-free methods for unconstrained nonsmooth optimization and its numerical analysis. Investigacao Operacional, 19, 75-93 (1999)

[Bag99b] Bagirov, A.M.: Minimization methods for one class of nonsmooth functions and calculation of semi-equilibrium prices. In: Eberhard. A. et al (eds) Progress in Optimization: Contribution from Australasia, 147-175. Kluwer Academic Publishers (1999)

[Bag02] A.M. Bagirov, A method for minimization of quasidifferentiable functions. Optimization Methods and Software, 17, 31-60 (2002)

[Bag05] Bagirov, A.M.: Max-min separability. Optimization Methods and Software, 20, 271-290 (2005)

[BRSY01] Bagirov, A.M., Rubinov, A., Soukhoroukova, N., Yearwood, J.: Unsupervised and supervised data classification via nonsmooth and global optimization. TOP, 11, 1-93, Sociedad de Estadistica Operativa, Madrid, Spain, June 2003 (2003)

[BRY00] Bagirov, A.M., Rubinov, A.M., Yearwood, J.: Using global optimization to improve classification for medical diagnosis and prognosis. Topics in Health Information Management, 22, 65-74 (2001)

[BRY02] Bagirov, A.M., Rubinov, A.M., Yearwood, J.: A global optimization approach to classification. Optimization and Engineering, 3, 129-155 (2002)

[BKS95] Bartels, S.G., Kuntz, L., Sholtes, S.: Continuous selections of linear functions and nonsmooth critical point theory. Nonlinear Analysis, TMA, 24, 385-407 (1995)

[BB97] Bennet, K.P., Blue, J.: A support vector machine approach to decision trees. Mathematics Report 97-100, Rensselaer Polytechnic Institute, Troy, New York (1997)

[BB96] Bennet, K.P., Bredersteiner, E.J.: A parametric optimization method for machine learning. INFORMS Journal on Computing, 9, 311-318, (1997)

[BM92] Bennett, K.P., Mangasarian, O.L.: Robust linear programming discrimination of two linearly inseparable sets. Optimization Methods and Software, 1, 23-34 (1992)

[BM93] Bennett, K.P., Mangasarian, O.L.: Bilinear separation of two sets in $n$ space. Computational Optimization and Applications, 2, 207-227 (1993)

[BM00] Bradley, P.S., Mangasarian, O.L.: Massive data discrimination via linear support vector machines. Optimization Methods and Software, 13, 1-10 (2000)

[BFM99] Bradley, P.S., Fayyad, U.M., O.L. Mangasarian: Data mining: overview and optimization opportunities. INFORMS Journal on Computing, 11, 217-238 (1999)

[Bur98] Burges, C.J.C.: A tutorial on support vector machines for pattern recognition. Data Mining and Knowledge Discovery, 2, 121-167 (1998)

[CM95] C. Chen, Mangasarian, O.L.: Hybrid misclassification minimization. Mathematical Programming Technical Report, 95-05, University of Wisconsin (1995)

[Cla83] Clarke, F.H.: Optimization and Nonsmooth Analysis, Wiley-Interscience, New York (1983) 
[DDM02] Demyanov, A.V., Demyanov, V.F., Malozemov, V.N.: Minmaxmin problems revisited. Optimization Methods and Software, 17, 783-804 (2002)

[DR95] Demyanov, V.F., Rubinov, A.M., Constructive Nonsmooth Analysis. Peter Lang, Frankfurt am Main (1995)

[Evt72] Evtushenko, Yu.G.: A numerical method for finding best guaranteed estimates. USSR Journal of Computational Mathematics and Mathematical Physics, 12, 109-128 (1972)

[HL93] Hiriart-Urruty, J.-B., Lemarechal, C.: Convex Analysis and Minimization Algorithms, Vol. 1 and Vol. 2. Springer Verlag, Berlin, Heidelberg, New York (1993)

[Kiw85] Kiwiel, K.C.: Methods of Descent for Nondifferentiable Optimization. Lecture Notes in Mathematics, 1133, Springer Verlag, Berlin (1985)

[Pol97] Polak, E.: Optimization: Algorithms and Consistent Approximations. Springer Verlag, New York (1997)

[KP98] Kirjner-Neto, C., Polak, E.: On the conversion of optimization problems with max-min constraints to standard optimization problems. SIAM J. Optimization, 8, 887-915 (1998)

[MN92] Makela, M.M., Neittaanmaki, P.: Nonsmooth Optimization. World Scientific, Singapore (1992)

[Man94] Mangasarian, O.L.: Misclassification minimization. Journal of Global Optimization, 5, 309-323 (1994)

[Man97] Mangasarian, O.L.: Mathematical programming in data mining. Data Mining and Knowledge Discovery, 1, 183-201 (1997)

[Mif77] Mifflin, R.: Semismooth and semiconvex functions in constrained optimization. SIAM Journal on Control and Optimization, 15, 957-972 (1977)

[MA92] Murphy, P.M., Aha, D.W.: UCI repository of machine learning databases. Technical report, Department of Information and Computer science, University of California, Irvine (1992) (www.ics.uci.edu/mlearn/MLRepository.html)

[NM65] Nelder, J.A., Mead, R.: A simplex method for function minimization. Comput. J., 7, 308-313 (1965)

[Pow02] Powell, M.J.D.: UOBYQA: unconstrained optimization by quadratic approximation. Mathematical Programming, Series B, 92, 555-582 (2002)

[Tho02] Thorsten, J.: Learning to Classify Text Using Support Vector Machines. Kluwer Academic Publishers, Dordrecht (2002)

[Vap95] Vapnik, V.N.: The Nature of Statistical Learning Theory. Springer, New York (1995) 